\title{
The use of fluorescence angiography to assess bowel viability in the acute setting: an international, multi-centre case series
}

\author{
Johanna J. Joosten ${ }^{1}$ (1) Grégoire Longchamp ${ }^{2} \cdot$ Mohammad F. Khan $^{3} \cdot$ Wytze Lameris $^{1}$. \\ Mark I. van Berge Henegouwen ${ }^{1}$. Wilhelmus A. Bemelman ${ }^{1}$ • Ronan A. Cahill ${ }^{3,4}$ - Roel Hompes ${ }^{1}$. Frédéric Ris ${ }^{2}$
}

Received: 8 October 2021 / Accepted: 13 February 2022 / Published online: 23 February 2022

(c) The Author(s) 2022

\begin{abstract}
Introduction Assessing bowel viability can be challenging during acute surgical procedures, especially regarding mesenteric ischaemia. Intraoperative fluorescence angiography (FA) may be a valuable tool for the surgeon to determine whether bowel resection is necessary and to define the most appropriate resection margins. The aim of this study is to report on FA use in the acute setting and to judge its impact on intraoperative decision making.

Materials and methods This is a multi-centre, retrospective case series of patients undergoing emergency abdominal surgery between February 2016 and 2021 in three general/colorectal units where intraoperative FA was performed to assess bowel viability. Primary endpoint was change of management after the FA assessment.

Results A total of 93 patients (50 males, $66.6 \pm 19.2$ years, ASA score $\geq$ III in $85 \%$ ) were identified and studied. Initial surgical approach was laparotomy in 66 (71\%) patients and laparoscopy in 27 (29\% and seven, 26\% conversions). The most common aetiologies were mesenteric ischaemia $(n=42,45 \%)$ and adhesional/herniae-related strangulation $(n=41,44 \%)$. In 50 patients a bowel resection was performed. Overall rates of anastomosis after resection, reoperation and 30-day mortality were $48 \%$ ( $n=24 / 50$, one leak), $12 \%$ and $18 \%$, respectively. FA changed management in 27 (29\%) patients. In four patients (4\% overall), resection was avoided and in 21 (23\%) extra bowel length was preserved (median $50 \mathrm{~cm}$ of bowel saved, IQR 28-98) although three patients developed further ischaemia. FA prompted extended resection (median of $20 \mathrm{~cm}$, IQR $10-50$ extra bowel) in six (6\%) patients.

Conclusion Intraoperative use of FA impacts surgical decisions regarding bowel resection for intestinal ischaemia, potentially enabling bowel preservation in approximately one out of four patients. Prospective studies are needed to optimize the best use of this technology for this indication and to determine standards for the interpretation of FA images and the potential subsequent need for second-look surgeries.
\end{abstract}

Keywords Fluorescence angiography $\cdot$ Acute setting $\cdot$ Change of management $\cdot$ Ischaemia $\cdot$ Case series

Intestinal ischaemia develops as a consequence of severe hypoperfusion caused by a variety of reasons which, if left

Roel Hompes

r.hompes@amsterdamumc.nl

1 Department of Surgery, Amsterdam University Medical Centres (UMC), University of Amsterdam, Cancer Centre Amsterdam, Meibergdreef 9, Amsterdam, the Netherlands

2 Division of Digestive Surgery, University Hospitals of Geneva, 1205 Geneva, Switzerland

3 Department of Surgery, Mater Misericordiae University, Hospital, 47 Eccles Street, Dublin 7, Ireland

4 UCD Centre for Precision Surgery, University College Dublin, Dublin, Ireland untreated, leads to transmural necrosis of the bowel wall followed by perforation, peritonitis, sepsis and organ failure. Even with treatment, this cascade results in high mortality rates exceeding $60 \%$ [1-3]. Patient's survival depends on prompt recognition and treatment to either achieve revascularization before ischaemia progresses to intestinal gangrene or resection of ischaemic segments of bowel. The incidence of treatable intestinal ischaemia appears to be rising, partly due to an increased awareness among clinicians but also an increasing incidence due to an ageing population surviving with severe cardiovascular or systemic disease [4].

At operation, determination of adequacy of bowel perfusion is essential and, where frank ischaemia is present, judgement of resection margins is vital. Extensive 
resections should be carefully considered, as removal of large segments of small bowel can result in short bowel syndrome (SBS) with intestinal failure. This is associated with poor quality of life and significant morbidity that increases with age [5]. If, however, the surgical approach is too conservative, and ischaemic bowel is left in situ, further clinical deterioration may result needing reoperation and increasing the risk of death. In the acute setting, judging the most appropriate resection extent may be difficult as a wide range of variables including haemodynamic instability and vasopressor support may co-exist. Also surgeon experience may be important [6]. Although many tools for intraoperative intestinal perfusion assessment have been considered over the years [7, 8], none have become standard due to their complexity and difficulty in reproducibility as well as their cost. A straight forward and useful intraoperative test would be very helpful.

Intraoperative, real time fluorescence angiography (FA) is a promising technique that has shown value for evaluation of adequate perfusion in gastrointestinal anastomoses in the elective setting $[9,10]$. Unfortunately, little is known about the application of FA in the acute setting [11]. Therefore, we aimed to report the impact of the use of FA in the acute setting on intraoperative decisions and clinical outcomes in three academic centres.

\section{Materials and methods}

We performed a retrospective analysis of a non-consecutive case series of all patients undergoing emergency surgery for bowel ischaemia in which FA was performed between February 2016 and 2021 in three tertiary referral centres. This study has been approved by the medical ethical committee of the Amsterdam University Medical Centres (AUMC)—location Amsterdam Medical Centre and has therefore been performed in accordance with the ethical standards laid down in the 1964 Declaration of Helsinki and its later amendments. This case series has been reported in line with the PROCESS Guideline [12]. Inclusion criteria were patients over 18 years old that underwent FA using indocyanine green (ICG) to assess intestinal perfusion during an emergency procedure for suspicion of bowel ischaemia. Patients were excluded when FA was performed after intestinal resection or with the sole purpose to assess perfusion of an anastomosis. Patient data with baseline characteristics, operative details and postoperative outcomes were retrospectively collected from the prospective maintained electronic patient systems of the different hospitals.

\section{FA procedure and endpoints}

All patients underwent acute surgical exploration, either via laparoscopy or laparotomy. In the case of mesenteric ischaemia, revascularization (if needed) was performed prior to fluorescence assessment. In all, intestinal perfusion appeared compromised (by macroscopical colourization of the bowel) and firstly the surgeon assessed the compromised segment of bowel by visual examination and determined a possible surgical plan; i.e. if bowel resection was necessary and if so the resection margins, subsequently FA assessment was performed by intravenous injection of a single bolus of ICG (Verdye, Diagnostic Green, Aschheim-Dornach, Germany). The definitive surgical plan based on the FA was then determined and carried out. Change of management due to $\mathrm{FA}(\mathrm{CoM}-\mathrm{FA})$ was defined as any deviation from the initial strategy determined by visual examination according to the result of the FA assessment. FA was performed with Stryker (Stryker, Kalamazoo, MI, USA.) imaging systems: either PINPOINT laparoscopic imaging system, Stryker 1688, or SPY Portable Handheld Imager (SPY-PHI). The primary outcome was CoM-FA. Secondary outcomes included length (in $\mathrm{cm}$ ) of additional bowel preserved or resected after FA, need for and number of reoperations, and mortality within 30 days. Reoperations were divided into planned (second look) and unplanned reoperations.

\section{Statistics}

All analyses were executed in IBM SPSS version 26 (IBM Corp. in Armonk, NY). The Shapiro-Wilk normality test was used to assess normal distribution. Data are expressed as mean and standard deviation (SD) for normally distributed continuous variables, median and interquartile range for non-normally distributed variables and proportions for binary variables.

\section{Results}

\section{Patient characteristics}

In total, 93 patients were included in the study with a mean age of 66.6 years at time of surgery (SD 19.2) and an American Society of Anaesthesiologists (ASA) score greater than two in $85 \%$. Of all patients, $50(54 \%)$ were male. The patient characteristics are outlined in Table 1 . The interventions were performed by 38 different surgeons with various level of surgical experience and more than half of the surgeries $(n=52 / 93)$ were performed by senior surgical trainees. 
Table 1 Patient characteristics

\begin{tabular}{llll}
\hline & Total & CoM-FA & No CoM-FA \\
\hline Total number of patients & 93 & 27 & 66 \\
$\quad$ Male & $50(54 \%)$ & $15(56 \%)$ & $35(53 \%)$ \\
Female & $43(46 \%)$ & $12(44 \%)$ & $31(47 \%)$ \\
Age in years, mean \pm SD & $66.6 \pm 19.2$ & $64.3 \pm 20.3$ & $67.6 \pm 18.8$ \\
ASA score & & & \\
I & 0 & 0 & 0 \\
II & $5(5 \%)$ & $2(7 \%)$ & $12(18 \%)$ \\
III & $53(57 \%)$ & $13(48 \%)$ & $40(61 \%)$ \\
IV & $26(28 \%)$ & $12(44 \%)$ & $14(21 \%)$ \\
Mean BMI, $\left(\mathrm{kg} / \mathrm{m}^{2}\right) \pm$ SD & $25.2 \pm 5.0$ & $25.1 \pm 4.4$ & $25.2 \pm 5.2$ \\
Cardiovascular history & $32(34 \%)$ & $10(37 \%)$ & $22(33 \%)$ \\
Diabetes & $22(24 \%)$ & $10(37 \%)$ & $12(18 \%)$ \\
\hline
\end{tabular}

CoM-FA change of management due to fluorescence angiography, ASA American Society of Anaesthesiology, BMI Body Mass Index

\section{Operation characteristics}

In the majority of cases $(n=66 / 93,71 \%)$, a laparotomy was performed. The remainder had their surgery commenced with laparoscopy with seven then needing conversion to laparotomy ( $26 \%$ conversion rate). Of laparotomies, $52 \%(n=34 / 66)$ were performed by consultants and $48 \%(n=32 / 66)$ by senior trainees while for laparoscopy the proportions were $35 \%(n=7 / 20)$ and $65 \%(n=13 / 20)$, respectively. All laparoscopic procedures $(n=7)$ which required conversion were carried out by senior trainees. The most common underlying aetiologies of ischaemia were mesenteric ischaemia in $45 \%(n=42 / 93)$ of patients and strangulation due to adhesion or internal herniation in $44 \%(n=41 / 93) .4 \%(n=4 / 93)$ were caused by a volvulus while the last $6 \%(n=6 / 93)$ concerned other causes: such as occlusive tumour and perforation. In 50 out of 93 patients, bowel resection was carried out (12 colonic, 24 small bowel and 14 both colonic and small bowel). Among them, $48 \%$ $(n=24 / 50)$ had an anastomosis constructed.

\section{CoM-FA}

FA resulted in a CoM in $29 \%$ of patients $(n=27 / 93)$. CoMFA led to either a more conservative or a more aggressive approach. The patient characteristics between these two groups are portrayed in Table 1 and outcomes overall as well as by aetiologies and COM in Table 2. Change to a more conservative approach occurred in 21 patients (Fig. 1, Table 2); in four of these patients $(n=4 / 21,19 \%)$, resection was avoided (Fig. 2) and in the remaining 17 (81\%), FA led to resection of a shorter segment of bowel. In this group, CoM-FA supporting a more conservative approach, a median of $50 \mathrm{~cm}$ of bowel (IQR 28-98) was preserved (for more detailed overview see Supplementary Table 1). In the CoM-FA group, FA assessment resulted in a more aggressive approach in six patients $(n=6 / 27)$, leading to a median of $20 \mathrm{~cm}$ (IQR 10-50) of additional resected bowel. The seniority of the surgeons was not decisive in the frequency of altering the surgical plan after FA; $52 \%$ of the cases in CoM-FA were by a senior surgeons versus $48 \%$ by a senior surgical trainee.

\section{Reoperations}

The overall 30-day reoperation rate was $12 \%(n=11)$ with four patients having progressive ischaemia (Table 3 ). In the group without a CoM-FA $(n=66 / 93)$, there was a reoperation rate of $8 \%(n=5 / 66)$, comprising four unplanned reoperations and one planned second look. One patient in this group had further bowel ischaemia identified (one of
Table 2 Outcomes by ischaemia aetiology and whether the fluorescence assessment changed management or not

\begin{tabular}{|c|c|c|c|c|}
\hline \multirow[t]{2}{*}{ Outcome } & \multirow{2}{*}{$\begin{array}{l}\text { No change of } \\
\text { management }\end{array}$} & \multicolumn{2}{|c|}{ Change of management } & \multirow[t]{2}{*}{ Total } \\
\hline & & $\begin{array}{l}\text { More conservative } \\
\text { approach }\end{array}$ & $\begin{array}{l}\text { More aggressive } \\
\text { approach }\end{array}$ & \\
\hline & $66(63 \%)$ & $21(23 \%)$ & $6(6 \%)$ & $93(100 \%)$ \\
\hline \multicolumn{5}{|l|}{ Aetiology ischaemia } \\
\hline Mesenteric ischaemia & $25(38 \%)$ & $12(57 \%)$ & $5(83 \%)$ & $42(45 \%)$ \\
\hline Strangulation & $32(48 \%)$ & $8(38 \%)$ & $1(17 \%)$ & $41(44 \%)$ \\
\hline Volvulus & $3(5 \%)$ & $1(5 \%)$ & 0 & $4(4 \%)$ \\
\hline Other & $6(9 \%)$ & 0 & 0 & $6(6 \%)$ \\
\hline $\begin{array}{l}\text { Bowel additionally preserved/ } \\
\text { resected in cm, median (IQR) }\end{array}$ & n.a. & $50(28-98)$ & $20(6-50)$ & n.a. \\
\hline Reoperation rate & $5(8 \%)$ & $6(29 \%)$ & 0 & $11(12 \%)$ \\
\hline Planned reoperations & $1(2 \%)$ & $4(19 \%)$ & 0 & $5(5 \%)$ \\
\hline Unplanned reoperations & $4(7 \%)$ & $2(10 \%)$ & 0 & $6(6 \%)$ \\
\hline Mortality & $10(15 \%)$ & $5(24 \%)$ & $2(33 \%)$ & $17(18 \%)$ \\
\hline
\end{tabular}




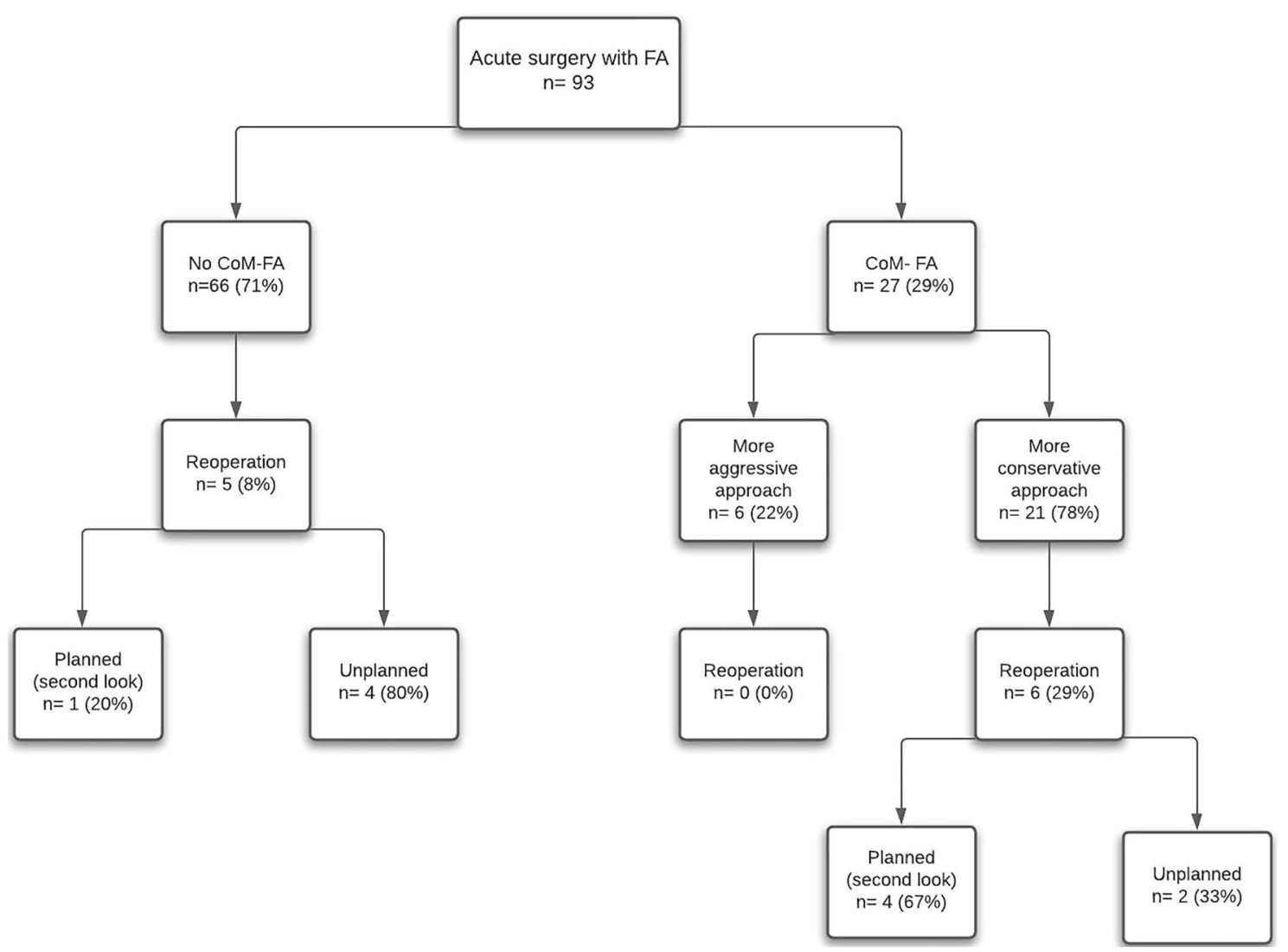

Fig. 1 Represents a flowchart of all patients with and without a change of management due to FA(CoM-FA/no CoM-FA) and the reoperation rates of the groups

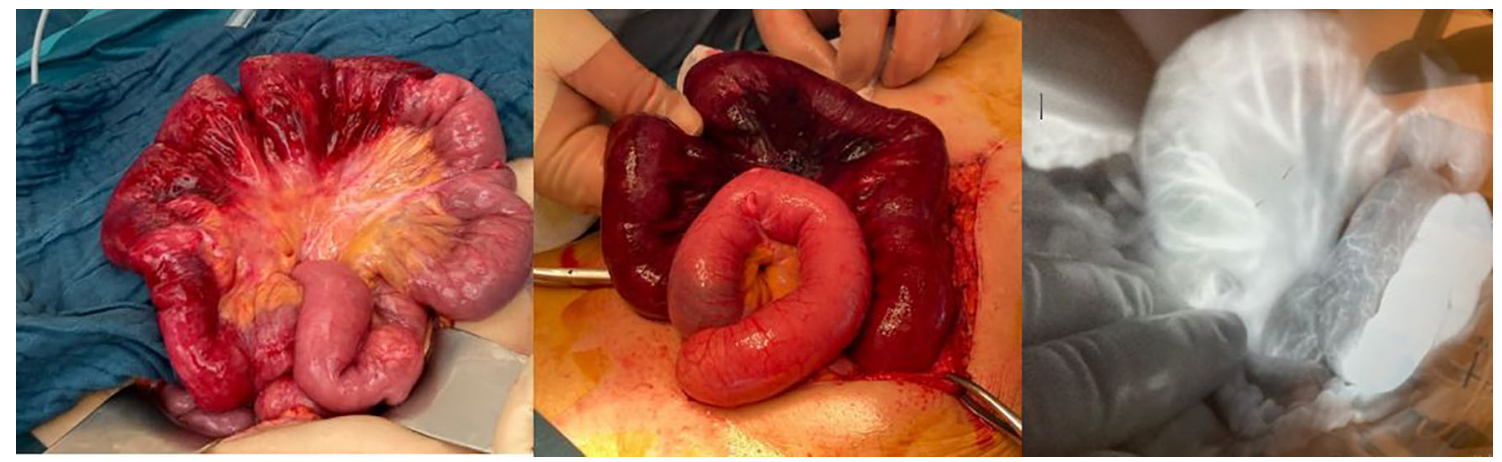

Fig. 2 Represents a case in which the perfusion was compromised in visual examination. FA assessment showed clear fluorescence enhancement, no resection was carried out. The patient had an uncomplicated postoperative course

the four unplanned reoperations). In the CoM-FA group, $22 \%(n=6 / 27)$ of the patients had a reoperation, all concerning patients with a more conservative approach after FA. Four planned (second look) surgeries (two of whom had further bowel ischaemia needing resection) and two unplanned operations (of whom one had further ischaemia). In patients who did not undergo a resection, 5\% $(n=2 / 43)$ needed a reoperation because of progressive ischaemia. Reoperation rates were 20\% $(n=8 / 41)$ for consultants and 6\% $(n=3 / 52)$ for senior surgical trainees. Regarding the surgical approach, $15 \%(n=10 / 66)$ of laparotomies and $4 \%(n=1 / 20)$ of laparoscopies required reoperation. An anastomotic leak occurred in one out of 24 patients (4\%) with an anastomosis with the diagnosis being made during a planned second-look surgery. 
Table 3 Reoperations specified per patient group

\begin{tabular}{|c|c|c|}
\hline Patient group & Unplanned reoperation & Planned reoperation (second look) \\
\hline No CoM-FA $(n=5 / 66)$ & $\begin{array}{l}\text { 1. Intra-abdominal bleeding query, no bleeding found } \\
\text { 2. Intra-abdominal bleeding query, additional resection of } \\
\text { ischaemic cecal pole } \\
\text { 3. Intra-abdominal lavage because of infected hematoma } \\
\text { 4. Intra-abdominal lavage because of infected hematoma }\end{array}$ & 1. Restoration of bowel continuity \\
\hline \multicolumn{3}{|l|}{ CoM-FA } \\
\hline More conservative approach $(n=6 / 27)$ & $\begin{array}{l}\text { 1. Evisceration and intra-peritoneal mesh placement } \\
\text { 2. Progressive ischaemia: } 30 \mathrm{~cm} \text { additional bowel resected }\end{array}$ & $\begin{array}{l}\text { 1. Negative second look } \\
\text { 2. Restoration of bowel continuity } \\
\text { 3. Progressive ischaemia: addi- } \\
\text { tional resection of } 50 \mathrm{~cm} \text { small } \\
\text { bowel } \\
\text { 4. Progressive ischaemia: addi- } \\
\text { tional resection of } 230 \mathrm{~cm} \text { small } \\
\text { bowel }\end{array}$ \\
\hline More aggressive approach $(n=0 / 6)$ & n.a. & n.a. \\
\hline
\end{tabular}

\section{Mortality}

In total, 17 patients died in this cohort, with an overall 30 -day mortality rate of $18 \%$ (Table 2). Mortality rates were $15 \%(n=10 / 66)$ in the no CoM-FA group, $24 \%$ $(n=5 / 21)$ in the group with a more conservative approach and $33 \%(n=2 / 6)$ with a more aggressive approach. In $76 \%$ ( $n=13 / 17$ ) of patients who died, the underlying aetiology was mesenteric ischaemia and in $24 \%(n=4 / 17)$ strangulation. Among the patients who died, 12 died due to sepsis and multi organ failure. Other causes of death were pneumonia $(n=2)$, cardiac arrest $(n=1)$ and liver failure $(n=1)$, acute haemorrhage after a thrombectomy of the superior mesenteric artery $(n=1)$. The mortality rate was higher in the laparotomy group: $23 \%(n=15 / 66)$, compared to the laparoscopic or conversion group $(5 \%, n=1 / 20), 14 \%(n=1 / 7)$, respectively. Among surgeries performed by consultants mortality rates were $27 \%(n=11 / 41)$ compared to $12 \%$ $(n=6 / 52)$ performed by senior surgical trainees.

\section{Discussion}

This international, multi-centre cohort study describes the use and outcomes of FA in the acute setting. Although FA has been widely implemented in the clinical setting for elective surgery, the potential added value in the acute setting has barely been studied. Among 93 operations for acute bowel ischaemia, a change of management was observed in $29 \%$, resulting in bowel preservation in approximately one out of four patients without a substantial increase in unplanned reoperations. The overall reoperation rate was $12 \%$ and the 30 -day mortality was $18 \%$, both of which are low compared to other published series [1-4, 13]. In half of the patients that underwent bowel resection, a primary anastomosis was made. In these patients, an acceptable leak rate of $4 \%$ was found.

This study also emphasizes that a median of $50 \mathrm{~cm}$ (IQR 28-98) of bowel could be spared among patients with a CoM-FA with a more conservative approach. Preserving $50 \mathrm{~cm}$ of small bowel or colon could make the difference in patients with extensive resections in preventing subsequent short bowel syndrome (which tends to occur when less of $100 \mathrm{~cm}$ of functioning bowel remains) [14].

The number of CoM-FA reported in this study corresponds with two prior studies investigating the use of FA in the acute setting; in both studies, FA provided additional information in $32-34.6 \%$ of the cases $[15,16]$. While definitive randomized control trials are currently ongoing [17], existing literature indicates that ICG use in vascularization assessment impacts the user's decision making in the elective setting in approximately $5-15 \%$ of cases [18, 19]. It seems therefore that FA assessments alter the surgical strategy more often in the acute than in the elective setting.

In our study, there was an overall reoperation rate of $12 \%$. This was $29 \%$ in patients in whom the CoM- FA encouraged preservation of bowel versus $8 \%$ in those in whom there was no CoM-FA. It's concerning that three patients in this group had further ischaemia identified and the mortality of this group was also higher than those in whom either no change or a more aggressive approach was followed. Some of the increased reoperation rate may be due to surgeons planning a second look with a lower threshold or to perform a delayed anastomosis after a more conservative approach is conceivable. Unplanned operation rates were similar between those without CoM-FA (7\%) as with those in whom CoM-FA preserved bowel (10\%) and in both groups, one patient was found to have progressive ischaemia. Three of the four cases requiring additional resection, however, took place in the conservative group, indicating that FA interpretation could 
be misleading and might require a proper learning curve to be proficient. As acute surgery is often performed out of office hours, the surgeon on call might have less experience using FA and interpret the FA differently than a more experienced user would, it has recently been demonstrated that there is a significant inter-observer variability of the interpretation of fluorescence imaging between expert and non-expert users in the elective setting [20]. Besides, in the acute setting, the surgeon has to contend with haemodynamic unstable patients with vasopressor requirement, which might result in difficult to interpret fluorescence images. When preserving bowel due to FA, there does seem to be a higher risk of progressive ischaemia and performing a planned reoperation (second look) at a lower threshold should be considered when preserving bowel.

The overall 30 -day mortality was $18 \%(n=17 / 93)$ in this series. If one looks at the mortality rates within the CoM-FA groups (Table 2), there is a higher mortality rate (24\% in conservative group and $33 \%$ in the more aggressive approach group), compared to the no CoM-FA group $(15 \%)$. This is noteworthy; however, this does not necessarily mean that this is related to the change of management itself. The CoM-FA groups have a different aetiology than the no change group, i.e. a higher proportion of mesenteric ischaemia, which is associated with a higher morbidity and mortality [3, 4]. Besides, in the CoM-FA group, there was a higher proportion of patients with an ASA score of IV; $44 \%$ versus $21 \%$ (Table 1 ). Also, it may be those who had CoM-FA had more complex ischaemic patterns to judge while those without were more discrete. It's noteworthy that consultant-case had higher mortality overall then those done by more junior staff, similarly suggesting differences other than this parameter alone.

While FA seems associated with positive outcomes in the overall group, the concerns identified in this study accentuate the need for further careful research including perhaps data-banking of the videos to allow understanding of the perfusion appearances and user interpretations. For instance, after reviewing FA images of patients who had progressive ischaemia during reoperation, two videos gave the impression of diffusion rather than adequate perfusion in the more conservative group and in our opinion should have been resected initially. This would also allow for training of surgeons without each having to acquire individual expert experience on the job. Furthermore quantification methods, with quantitative values that define a threshold for adequate perfusion, may help standardize interpretations. In addition, protocolized approaches are needed to be developed. From our own experience, endorsed by this study, we suggest that perfusion judgement should be determined within the first minute as, even though the bowel may fluoresce later, this may be falsely positive occurring due to diffusion of ICG into the tissue rather than true perfusion [21].
This study was limited by its retrospective nature. However, CoM-FA was reported in the operation reports prospectively. Yet, due to the retrospective nature of this study, we could not distinguish between the different kinds of aetiology of non-adhesional and non-volvulus bowel ischaemia (i.e. whether low flow, thrombotic disease, non-occlusive mesenteric ischaemia). In addition, quantitative values such as time to fluorescence were not captured. Besides, patients were not consecutively included, which may have resulted in a selection bias. There is also a lack of contemporaneous control data to give context to the clinical outcomes here reported. Prospective studies which both include quantitative fluorescence values as well as differentiate between the different manifestations of intestinal ischaemia are needed.

In conclusion, this study, the largest multi-centre case series yet, presents the use of FA in the acute setting of patients operated on because of ischaemia. Results from this study support that FA can provide useful, additional information besides visual evaluation alone on a reproducible manner. However, prospective studies are needed to optimize the best use of this technology for this indication and to determine standards for the interpretation of FA images and the potential subsequent need for second-look surgeries.

Supplementary Information The online version contains supplementary material available at https://doi.org/10.1007/s00464-022-09136-7.

Acknowledgements Not applicable.

Funding This paper was not funded.

\section{Declarations}

Disclosures Prof. Dr. M. I. van Berge Henegouwen: unrestricted grants from Olympus and Stryker and consultancy for Johnson and Johnson Alesi, Surgical, Mylan, Braun, and Medtronic. All fees and grants paid to institution. Prof. Dr. W. A. Bemelman: speaker fees from Johnson \& Johnson, Medtronic, Takeda, consulting fees from Takeda and Braun. Prof. Dr. R. Cahill; speaker fees from Stryker Corp, Olympus and Ethicon/J\&J, research funding from Intuitive Corp and Medtronic and from the Irish Government (DTIF) in collaboration with IBM Research in Ireland and from EU Horizon 2020 in collaboration with Palliare. Dr. R. Hompes: unrestricted grant and materials Stryker European Operations B.V. Prof. Dr. F. Ris: Research grant from Quantgene and Johnson \& Johnson, consulting for Arthrex, Distal Motion, Hollister, Freka, and Stryker. Dr. J. J. Joosten, Dr. W. Lameris, Dr. G. Longchamp, and Dr. M. F. Khan have no conflict or financial ties to disclose.

Open Access This article is licensed under a Creative Commons Attribution 4.0 International License, which permits use, sharing, adaptation, distribution and reproduction in any medium or format, as long as you give appropriate credit to the original author(s) and the source, provide a link to the Creative Commons licence, and indicate if changes were made. The images or other third party material in this article are included in the article's Creative Commons licence, unless indicated otherwise in a credit line to the material. If material is not included in the article's Creative Commons licence and your intended use is not permitted by statutory regulation or exceeds the permitted use, you will 
need to obtain permission directly from the copyright holder. To view a copy of this licence, visit http://creativecommons.org/licenses/by/4.0/.

\section{References}

1. Stoney RJ, Cunningham CG (1993) Acute mesenteric ischemia. Surgery 114(3):489-490

2. Oldenburg WA, Lau LL, Rodenberg TJ, Edmonds HJ, Burger CD (2004) Acute mesenteric ischemia: a clinical review. Arch Intern Med 164(10):1054-1062. https://doi.org/10.1001/archinte.164.10. 1054

3. Leone $\mathrm{M}$ et al (2015) Outcome of acute mesenteric ischemia in the intensive care unit: a retrospective, multicenter study of 780 cases. Intensive Care Med 41(4):667-676. https://doi.org/10.1007/ s00134-015-3690-8

4. McKinsey JF, Gewertz BL (1997) Acute mesenteric ischemia. Surg Clin North Am 77(2):307-318. https://doi.org/10.1016/ s0039-6109(05)70550-8

5. Tilsed JV et al (2016) ESTES guidelines: acute mesenteric ischaemia. Eur J Trauma Emerg Surg 42(2):253-270. https://doi.org/10. 1007/s00068-016-0634-0

6. Schuster KM, Hazelton JP, Rattigan D, Perez JM, Bhattacharya B (2021) Association of acute care surgeon experience with emergency surgery patient outcomes and mortality. JAMA Surg 156(5):472-478. https://doi.org/10.1001/jamasurg.2021.0041

7. Danse EM, Van Beers BE, Goffette P, Dardenne AN, Laterre PF, Pringot J (1996) Diagnosis of acute intestinal ischemia by color Doppler sonography. Color Doppler sonography and acute intestinal ischemia. Acta Gastroenterol Belg 59(2):140-142

8. Singh DB, Stansby G, Bain I, Harrison DK (2009) Intraoperative measurement of colonic oxygenation during bowel resection. Adv Exp Med Biol 645:261-266. https://doi.org/10.1007/978-0-38785998-9_39

9. Slooter MD, Eshuis WJ, Cuesta MA, Gisbertz SS, van Berge Henegouwen MI (2019) Fluorescent imaging using indocyanine green during esophagectomy to prevent surgical morbidity: a systematic review and meta-analysis. J Thorac Dis. https://doi.org/ 10.21037/jtd.2019.01.30

10. Schaafsma BE et al (2011) "The clinical use of indocyanine green as a near-infrared fluorescent contrast agent for image-guided oncologic surgery," (in Eng). J Surg Oncol 104(3):323-332. https://doi.org/10.1002/jso.21943

11. Liot $\mathrm{E}$ et al (2018) Does near-infrared (NIR) fluorescence angiography modify operative strategy during emergency procedures?
Surg Endosc 32(10):4351-4356. https://doi.org/10.1007/ s00464-018-6226-9

12. Agha RA et al (2020) The PROCESS 2020 guideline: updating consensus preferred reporting of CasESeries in surgery (PROCESS) guidelines. Int J Surg (London, England) 84:231-235. https://doi.org/10.1016/j.ijsu.2020.11.005

13. Nielsen LBJ, Tengberg LT, Bay-Nielsen M (2017) Laparoscopy in major abdominal emergency surgery seems to be a safe procedure. Dan Med J 64(5):15370

14. Group EIF et al (2016) European Society of Coloproctology consensus on the surgical management of intestinal failure in adults. Colorectal Dis 18(6):535-48. https://doi.org/10.1111/codi.13321

15. Liot E et al (2018) "Does near-infrared (NIR) fluorescence angiography modify operative strategy during emergency procedures?," (in Eng). Surg Endosc. https://doi.org/10.1007/ s00464-018-6226-9

16. Karampinis I et al (2018) "Indocyanine green tissue angiography can reduce extended bowel resections in acute mesenteric ischemia," (in Eng). J Gastrointest Surg. https://doi.org/10.1007/ s11605-018-3855-1

17. Armstrong G et al (2018) "IntAct: intra-operative fluorescence angiography to prevent anastomotic leak in rectal cancer surgery: a randomized controlled trial," (in Eng). Colorectal Dis. https:// doi.org/10.1111/codi.14257

18. Shen R, Zhang Y, Wang T (2018) Indocyanine green fluorescence angiography and the incidence of anastomotic leak after colorectal resection for colorectal cancer: a meta-analysis. Dis Colon Rectum 61(10):1228-1234. https://doi.org/10.1097/DCR.0000000000 001123

19. Ris F et al (2014) "Near-infrared (NIR) perfusion angiography in minimally invasive colorectal surgery," (in Eng). Surg Endosc 28(7):2221-2226. https://doi.org/10.1007/s00464-014-3432-y

20. Hardy NP, Dalli J, Khan MF, Andrejevic P, Neary PM, Cahill RA (2021) Inter-user variation in the interpretation of near infrared perfusion imaging using indocyanine green in colorectal surgery. Surg Endosc. https://doi.org/10.1007/s00464-020-08223-x

21. Dalli J, Hardy N, Mac Aonghusa PG, Epperlein JP, Cantillon Murphy P, Cahill RA (2021) Challenges in the interpretation of colorectal indocyanine green fluorescence angiography-a video vignette. Colorectal Dis 23(5):1289-1290. https://doi.org/10. $1111 /$ codi. 15592

Publisher's Note Springer Nature remains neutral with regard to jurisdictional claims in published maps and institutional affiliations. 